\title{
An Adequate Representation of Medical Data Based on Partial Set Approximation
}

\author{
Zoltán Ernő Csajbók ${ }^{1}$, Tamás Mihálydeák ${ }^{2}$, and József Ködmön ${ }^{1}$ \\ 1 Department of Health Informatics, Faculty of Health, University of Debrecen, \\ Sóstói út 2-4, H-4400 Nyíregyháza, Hungary \\ \{csajbok.zoltan, kodmon.jozsef\}@foh.unideb.hu \\ 2 Department of Computer Science, Faculty of Informatics, University of Debrecen \\ Kassai út 26, H-4028 Debrecen, Hungary \\ mihalydeak. tamas@inf .unideb.hu
}

\begin{abstract}
Computer aided medical diagnosis and treatment require an adequate representation of uncertain or imperfect medical data. There are many approaches dealing with such type of data. Pawlak proposed a new method called rough set theory. In this paper, beyond classical and recent methods, the authors propose a basically new approach. It relies on a generalization of rough set theory, namely, the partial covering of the universe of objects. It adequately reflects the partial nature of real-life problems. This new approach called the partial approximation of sets is presented as well as its medical informatics application is demonstrated.
\end{abstract}

Keywords: Rough set theory, partial approximation of sets, tool-based approximation framework, thyroid disease diagnosis.

\section{Introduction}

Uncertainty, imprecision or incompleteness are important aspects of patients' medical data. Therefore, it is an inevitable challenge of medical informatics how imperfect medical data can be represented, moreover, how appropriate and powerful approximative inference methods can be provided. There are two classical approaches, Bayesian statistics [1, 25] and Dempster-Shafer theory of evidence [2, 28]. In the middle of 1960s, Zadeh proposed fuzzy set theory [22, 23, 30]. The medical expert system MYCIN uses the so-called certainty factor (CF) model [4]. Recently knowledge-based systems and other artificial intelligence methods have also been used in the medical diagnosis and treatment [17, 18].

In the early 1980s, Pawlak proposed a new method called rough set theory [19, 20]. It can be viewed as a new mathematical approach to manage imperfect or vague knowledge [15, 12]. It has extensive applications, among others, in artificial intelligence, cognitive sciences and medicine [11, 26]. Its starting point is a nonempty finite set $U$ of objects and an equivalence relation $\varepsilon$ on $U$ [20]. The equivalence classes are called $\varepsilon$-elementary sets. They can be viewed as sets of indiscernible objects characterized by the same available information about them [21, 24]. Definable sets are any unions of $\varepsilon$-elementary sets. 
Then, any set $S \subseteq U$ can be naturally approximated by the so-called lower and upper $\varepsilon$-approximations of $S$. The former is the union of all $\varepsilon$-elementary sets which are the subsets of $S$, whereas the latter is the union of all $\varepsilon$-elementary sets which have a nonempty intersection with $S$.

In rough set theory, the equivalence classes are pairwise disjoint and cover the universe. Giving up the disjoint property but retaining the covering, a natural generalization of rough set theory is obtained. It is called covering-based rough set theory [3, 29, 31 33. The partial nature of real-life problems, however, requires working out partial approximation schemes. Thus, not only the pairwise disjoint property but also the covering of the universe are given up. This basically new approach is referred to as partial approximation of sets [5, 6, 10].

Furthermore, based on partial approximation of sets, a general tool-based approximation framework can be built up [9]. It is a general model which has applications in computer security [7, 8]. In this paper, we apply it to representing imperfect medical data.

The paper is organized as follows. Having reviewed the fundamental notions of partial approximation of sets in Section 2, Section 3 presents the general toolbased approximation framework. Section 4 shows a simplified thyroid disease diagnosis example in order to demonstrate how our approach can be applied. Finally, in Section 5, we conclude the paper.

\section{Fundamental Notions of Partial Approximation of Sets}

A general set-theoretic approximation framework has four components:

- the domain of approximations whose sets are approximated;

- some distinguished sets of the domain as the basis of approximations;

- definable sets deriving from base sets in some way or other as possible approximations of sets of the domain;

- an approximation pair determining the lower and upper approximations of sets using definable sets.

Definable sets represent our available knowledge about the domain. They can be thought of as tools of the approximation process and simply called tools. In particular, base sets can be viewed as primary tools, definable sets as derived tools. The way of getting derived tools from primary tools shows how primary tools are used. An approximation pair prescribes the utilization of primary and derived tools in a whole approximation process.

Let $U$ be a finite nonempty set.

Let $\mathfrak{B} \subseteq 2^{U}$ be a nonempty family of nonempty subsets of $U \mathbb{1} \mathfrak{B}$ is called the base system, its members are the base sets. Let $\mathfrak{D}_{\mathfrak{B}} \subseteq 2^{U}$ denote an extension of $\mathfrak{B}$ in such a way that 1) $\mathfrak{B} \subseteq \mathfrak{D}_{\mathfrak{B}}$, and 2) $\emptyset \in \mathfrak{D}_{\mathfrak{B}}$. The members of $\mathfrak{D}_{\mathfrak{B}}$ are called definable sets. It is not necessary that $\bigcup \mathfrak{D}_{\mathfrak{B}}=U$. An approximation framework is total if $\bigcup \mathfrak{D}_{\mathfrak{B}}=U$, and it is partial otherwise.

\footnotetext{
${ }^{1} 2^{U}$ denotes the power set of $U$.
} 
It is a natural assumption that $\mathfrak{D}_{\mathfrak{B}}$ is obtained (derived) form $\mathfrak{B}$ by some sort of set type transformations (for the most important cases, see [5]). In order to build a generalized Pawlakian partial approximation framework, we define $\mathfrak{D}_{\mathfrak{B}}$ with the following definition:

1. $\emptyset \in \mathfrak{D}_{\mathfrak{B}} ;$

2. $\mathfrak{B} \subseteq \mathfrak{D}_{\mathfrak{B}}$;

3. if $\overline{\mathfrak{B}}^{\prime} \subseteq \mathfrak{B}, \cup \mathfrak{B}^{\prime}, \cap \mathfrak{B}^{\prime} \in \mathfrak{D}_{\mathfrak{B}}$.

Next, let $\langle\mathrm{I}, \mathrm{u}\rangle$ be an ordered pair of maps I, u : $2^{U} \rightarrow 2^{U}$ on $\left(2^{U}, \subseteq\right)$. Of course, their intended meaning is to express the lower and upper approximations of any sets with the help of the beforehand given definable sets as tools. Hence, $\langle\mathrm{I}, \mathrm{u}\rangle$ is called an approximation pair.

Here, for any $S \subseteq U$, the lower and upper approximations are a possible generalization of Pawlakian lower and upper $\varepsilon$-approximations:

- lower approximation $\mathrm{I}(S)$ is the union of all definable sets which are the subsets of $S$ :

$$
\mathrm{I}(S)=\bigcup\left\{D \in \mathfrak{D}_{\mathfrak{B}} \mid D \subseteq S\right\} \in \mathfrak{D}_{\mathfrak{B}}
$$

- upper approximation $\mathrm{u}(S)$ is the union of all definable sets which have a nonempty intersection with $S$ :

$$
\mathrm{u}(S)=\bigcup\left\{D \in \mathfrak{D}_{\mathfrak{B}} \mid D \cap S \neq \emptyset\right\} \in \mathfrak{D}_{\mathfrak{B}} .
$$

The universe $U$, the base system $\mathfrak{B}$, the set $\mathfrak{D}_{\mathfrak{B}}$ of definable sets, and the approximation pair $\langle\mathbf{I}, \mathbf{u}\rangle$ together are called the partial approximation space and denoted by the ordered 5 -tuple $\left\langle 2^{U}, \mathfrak{B}, \mathfrak{D}_{\mathfrak{B}},\left.\right|_{\mathfrak{B}}, \mathbf{u}_{\mathfrak{B}}\right\rangle$.

Possible interpretations of lower and upper approximations of a set $S \subseteq U$ are the following [20]:

1. I $(S)$ is the set of all members of $U$ which can certainly be classified as belonging to $S$ with respect to $\mathfrak{B}$ (positive region);

2. $\mathrm{u}(S)$ is the set of all members of $U$ which can possibly be classified as belonging to $S$ with respect to $\mathfrak{B}$;

3. $U \backslash \mathrm{u}(S)$ is the set of all members of $U$ which can certainly be classified as not belonging to $S$ with respect to $\mathfrak{B}$ (negative region).

\section{Elements of Tool-Based Approximation Framework}

Tool-based approximation framework is defined relying on partial approximation spaces. For more details, see [9].

Let $A^{+}, A^{-} \subseteq U$ be two nonempty subsets of $U$ in such a way that $A^{+} \cap A^{-}=\emptyset . A^{+}$and $A^{-}$are called the positive and negative reference set, respectively. The adjectives "positive" and "negative" claim nothing else but that the sets $A^{+}$and $A^{-}$are well separated.

Furthermore, let $\mathfrak{T}^{+}, \mathfrak{T}^{-} \subseteq 2^{U}$ be two nonempty finite families of nonempty subsets of $U$, where $\mathfrak{T}^{+}$and $\mathfrak{T}^{-}$are not necessarily disjoint. The members of $\mathfrak{T}^{+}$ are called positive or $\mathfrak{T}^{+}$-tools, whereas the members of $\mathfrak{T}^{-}$are called negative or $\mathfrak{T}^{-}$-tools. 
Requirements for positive and negative tools are the following:

- sets in $\mathfrak{T}^{+}\left(\mathfrak{T}^{-}\right)$are not necessarily pairwise disjoint;

- neither $\bigcup \mathfrak{T}^{+}$nor $\bigcup \mathfrak{T}^{-}$covers $U$ necessarily;

- for each subset $T^{+} \in \mathfrak{T}^{+}\left(T^{-} \in \mathfrak{T}^{-}\right)$, it is easy to decide whether a member of $U$ belongs to $T^{+}\left(T^{-}\right)$or does not.

Mutual relationship between positive/negative tools and positive/negative reference sets may provide useful information. They can be obtained with the help of partial approximation of positive/negative reference sets within the approximation spaces $\left\langle 2^{U}, \mathfrak{T}^{+}, \mathfrak{D}_{\mathfrak{T}^{+}}, \mathrm{I}_{\mathfrak{T}^{+}}, \mathbf{u}_{\mathfrak{T}^{+}}\right\rangle,\left\langle 2^{U}, \mathfrak{T}^{-}, \mathfrak{D}_{\mathfrak{T}^{-}}, \mathrm{I}_{\mathfrak{T}^{-}}, \mathbf{u}_{\mathfrak{T}^{-}}\right\rangle$, respectively.

\section{Thyroid Disease Diagnosis Support}

Let us suppose that we study the symptoms of the thyroid dysfunction [14]. Clinical symptoms which manifest thyroid dysfunction progress slowly they are often nonspecific and could represent other not thyroid disorders. Hence, thyroid function diagnosis via clinical symptoms is an important but inexact classification problem [27, 16].

For the sake of simplicity, we deal with only hypothyroidism and hyperthyroidism thyroid disorders [13]. The thyroid gland produces thyroid hormones, thyroxine (T4) and triiodothyronine (T3). Hyperthyroidism occurs when the thyroid is "overactive", i.e., releases too much hormones, whereas hypothyroidism takes place when the thyroid is "underactive", i.e., does not produce enough hormones.

We have at our disposal an information table (Table 10) containing clinical symptoms which may indicate whether hypothyroidism or hyperthyroidism, or else neither of the two. There are, of course, more symptoms of hypothyroidism and hyperthyroidism, but we have simplified the example here for illustrative purposes.

Table 1. Clinical symptoms of thyroid dysfunction and diagnosis based on test results

\begin{tabular}{|c|c|c|c|c|c|c|c|}
\hline No. & $\begin{array}{c}\text { Weight } \\
\text { change }\end{array}$ & Edema & $\begin{array}{c}\text { Tachy- } \\
\text { cardia }\end{array}$ & $\begin{array}{c}\text { Increased } \\
\text { sweating }\end{array}$ & Affection & $\begin{array}{c}\text { Hypothy- } \\
\text { roidism }\end{array}$ & $\begin{array}{c}\text { Hyperthy- } \\
\text { roidism }\end{array}$ \\
\hline$P_{1}$ & not change & no & no & no & normal & no & no \\
\hline$P_{2}$ & gain & no & no & no & normal & yes & no \\
\hline$P_{3}$ & gain & no & yes & no & normal & yes & no \\
\hline$P_{4}$ & loss & no & yes & yes & normal & no & yes \\
\hline$P_{5}$ & not change & yes & no & yes & nervousness & no & yes \\
\hline
\end{tabular}

The universe of objects is the set of patients, i.e., $U=\left\{P_{1}, P_{2}, P_{3}, P_{4}, P_{5}\right\}$.

Hypothyroidism and hyperthyroidism can be accurately diagnosed with laboratory tests. Last two columns in Table 1 are based on these results. Reference sets are formed on them: the "positive" reference set is: $A^{\text {Hypo }}=\left\{P_{2}, P_{3}\right\}$, and the "negative" reference set is: $A^{\text {Hyper }}=\left\{P_{4}, P_{5}\right\}$. 
Note that $A^{\text {Hypo }} \cap A^{\text {Hyper }}=\emptyset$.

Table 2 contains clinical symptoms which may indicate hypothyroidism ("positive tools") and hyperthyroidism ("negative tools"). Notice that $\mathfrak{T}^{\text {Hypo and }}$ $\mathfrak{T}^{\text {Hyper }}$ do not necessarily cover the universe $\left(\bigcup \mathfrak{T}^{\text {Hypo }}, \bigcup \mathfrak{T}^{\text {Hyper }} \subseteq U\right)$ and their members are not pairwise disjoint, respectively.

Table 2. Symptoms which may indicate hypothyroidism/hyperthyroidism ("positive" / "negative" tools)

\begin{tabular}{|l|l|l|l|}
\hline \multicolumn{2}{|l|}{ Symptoms which may indicate hypothyroidism } & \multicolumn{1}{|c|}{ Symptoms which may indicate hyperthyroidism } \\
\hline Weight change $=$ gain & $T_{1}^{\text {Hypo }}=\left\{P_{2}, P_{3}\right\}$ & Weight change $=$ loss & $T_{1}^{\text {Hyper }}=\left\{P_{4}\right\}$ \\
\hline Edema = yes & $T_{2}^{\text {Hypo }}=\left\{P_{5}\right\}$ & Tachycardia = yes & $T_{2}^{\text {Hyper }}=\left\{P_{3}, P_{4}\right\}$ \\
\hline Tachycardia = yes & $T_{3}^{\text {Hypo }}=\left\{P_{3}, P_{4}\right\}$ & Increased sweating $=$ yes & $T_{3}^{\text {Hyper }}=\left\{P_{4}, P_{5}\right\}$ \\
\hline Affection $=$ depression & $\emptyset$ & Affection $=$ nervousness & $T_{4}^{\text {Hyper }}=\left\{P_{5}\right\}$ \\
\hline \multicolumn{2}{|c|}{$\tau^{\text {Hypo }}=\left\{T_{1}^{\text {Hypo }}, T_{2}^{\text {Hypo }}, T_{3}^{\text {Hypo }}\right\}$} & $\mathfrak{T}^{\text {Hyper }}=\left\{T_{1}^{\text {Hyper }}, T_{2}^{\text {Hyper }}, T_{3}^{\text {Hyper }}, T_{4}^{\text {Hyper }}\right\}$ \\
\hline
\end{tabular}

Primary tools are (see Table 2):

$-\mathfrak{T}^{\text {Hypo }}=\left\{T_{1}^{\text {Hypo }}, T_{2}^{\text {Hypo }}, T_{3}^{\text {Hypo }}\right\}=\left\{\left\{P_{2}, P_{3}\right\},\left\{P_{5}\right\},\left\{P_{3}, P_{4}\right\}\right\} ;$

$-\mathfrak{T}^{\text {Hyper }}=\left\{T_{1}^{\text {Hyper }}, T_{2}^{\text {Hyper }}, T_{3}^{\text {Hyper }}, T_{4}^{\text {Hyper }}\right\}=\left\{\left\{P_{4}\right\},\left\{P_{3}, P_{4}\right\},\left\{P_{4}, P_{5}\right\},\left\{P_{5}\right\}\right\}$.

Derived tools $\mathfrak{D}_{\mathfrak{T} \text { H ypo }}$ and $\mathfrak{D}_{\mathfrak{T} H \text { yper }}$ are formed by unions and intersections of any subsets of $\mathfrak{T}^{\text {Hypo }}$ and $\mathfrak{T}^{\text {Hyper }}$, respectively:

$$
\begin{aligned}
\mathfrak{D}_{\mathfrak{T} \text { Hypo }}= & \left\{\emptyset, T_{1}^{\text {Hypo }}, T_{2}^{\text {Hypo }}, T_{3}^{\text {Hypo }},\right. \\
& \left.\left\{P_{2}, P_{3}, P_{5}\right\},\left\{P_{2}, P_{3}, P_{4}\right\},\left\{P_{3}, P_{4}, P_{5}\right\},\left\{P_{2}, P_{3}, P_{4}, P_{5}\right\},\left\{P_{3}\right\}\right\} ; \\
\mathfrak{D}_{\mathfrak{T}_{\text {Hyper }}}= & \left\{\emptyset, T_{1}^{\text {Hyper }}, T_{2}^{\text {Hyper }}, T_{3}^{\text {Hyper }}, T_{4}^{\text {Hyper }},\right. \\
& \left.\left\{P_{3}, P_{4}, P_{5}\right\},\left\{P_{4}\right\},\left\{P_{5}\right\}\right\} .
\end{aligned}
$$

Sample evaluations can be carried out as follows.

Let us take, e.g., the group of patients having hypothyroidism diagnosis: $A^{\text {Нуро }}=\left\{P_{2}, P_{3}\right\} \subseteq U$.

Table 3. Approximating hypothyroidism with clinical symptoms which may indicate hypothyroidism itself

\begin{tabular}{|c|c|c|c|c|c|c|c|}
\hline No. & Weight change & $\begin{array}{c}\text { Edem } \\
\text { a }\end{array}$ & $\begin{array}{c}\text { Tachy- } \\
\text { cardia }\end{array}$ & $\begin{array}{c}\text { Increased } \\
\text { sweating }\end{array}$ & Affection & $\begin{array}{c}\text { Hypothy- } \\
\text { roidism }\end{array}$ & $\begin{array}{c}\text { Hyperthy- } \\
\text { roidism }\end{array}$ \\
\hline$P_{1}$ & not change & no & no & no & normal & no & no \\
\hline$P_{2}$ & gain & no & no & no & normal & yes & no \\
\hline$P_{3}$ & gain & gon & yes & no & normal & yes & no \\
\hline$P_{4}$ & loss & no & yes & yes & normal & no & yes \\
\hline$P_{5}$ & not change & yes & no & yes & nervousness & no & yes \\
\hline
\end{tabular}


The evaluation of patients having hypothyroidism with respect to the clinical symptoms which may indicate hypothyroidism, i.e., in the language of partial ap-

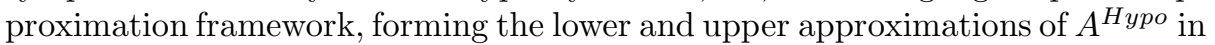
the partial approximation space $\left\langle 2^{U}, \mathfrak{T}^{H y p o}, \mathfrak{D}_{\mathfrak{T} H y p o},\left.\right|_{\mathfrak{T} H y p o}, \mathbf{u}_{\mathfrak{T} H y p o}\right\rangle$ (see Table 3):

$-\mathrm{I}_{\mathfrak{T} \text { Hypo }}\left(A^{\text {Hypo }}\right)=\mathrm{I}_{\mathfrak{T} \text { Hypo }}\left(\left\{P_{2}, P_{3}\right\}\right)=T_{1}^{\text {Hypo }} \cup\left(T_{1}^{\text {Hypo }} \cap T_{3}^{\text {Hypo }}\right)=\left\{P_{2}, P_{3}\right\}$. Informally: patients having "weight gain" or "weight gain and tachycardia" can certainly be classified as suffering from hypothyroidism with respect to the clinical symptoms which may indicate hypothyroidism.

$-\mathrm{u}_{\mathfrak{T}_{\text {H } \text { yро }}}\left(A^{\text {Hypo }}\right)=\mathrm{u}_{\mathfrak{T}_{\text {H ypo }}}\left(\left\{P_{2}, P_{3}\right\}\right)=T_{1}^{\text {Hypo }} \cup T_{3}^{\text {Hypo }} \cup\left(T_{1}^{\text {Hypo }} \cap T_{3}^{\text {Hypo }}\right)=$ $\left\{P_{2}, P_{3}, P_{4}\right\}$.

Informally: patients having "weight gain" or "tachycardia" or "weight gain and tachycardia" can possibly be classified as suffering from hypothyroidism with respect to the clinical symptoms which may indicate hypothyroidism.

Table 4. Approximating hypothyroidism with clinical symptoms which may indicate hyperthyroidism

\begin{tabular}{|c|c|c|c|c|c|c|c|}
\hline No. & Weight change & $\begin{array}{c}\text { Edem } \\
\text { a }\end{array}$ & $\begin{array}{l}\text { Tachy- } \\
\text { cardia }\end{array}$ & $\begin{array}{c}\text { Increased } \\
\text { sweating }\end{array}$ & Affection & \begin{tabular}{|c|}
$\begin{array}{c}\text { Hypothy- } \\
\text { roidism }\end{array}$ \\
\end{tabular} & $\begin{array}{c}\text { Hyperthy- } \\
\text { roidism }\end{array}$ \\
\hline$P_{1}$ & not change & no & no & no & normal & no & no \\
\hline$P_{2}$ & gain & no & no & 110 & normal & yes & no \\
\hline$P_{3}$ & gain & no & yes & 190 & mormal & yes & no \\
\hline$P_{4}$ & loss & no & yes & yes & normal & no & yes \\
\hline$P_{5}$ & not change & yes & no & yes & nervousmess & no & yes \\
\hline
\end{tabular}

The evaluation of patients having hypothyroidism with respect to the clinical symptoms which may indicate hyperthyroidism, i.e., in the language of partial approximation framework, forming the lower and upper approximations of $A^{\text {Hypo }}$ in the partial approximation space $\left\langle 2^{U}, \mathfrak{T}^{\text {Hyper }}, \mathfrak{D}_{\mathfrak{T} H \text { yper }},\left.\right|_{\mathfrak{T} H \text { yper }}, \mathbf{u}_{\mathfrak{T} H \text { yper }}\right\rangle$ (see Table 4) (it can be viewed as a "cross-evaluation"):

$-\mathrm{I}_{\mathfrak{T} \text { Hyper }}\left(A^{\text {Hypo }}\right)=\mathrm{I}_{\mathfrak{T} \text { Hyper }}\left(\left\{P_{2}, P_{3}\right\}\right)=\emptyset$.

Informally: in accordance with the expectations, there are no symptoms under which patients can certainly be classified as suffering from hypothyroidism with respect to the clinical symptoms which may indicate hyperthyroidism.

$-\mathrm{u}_{\mathfrak{T}_{\text {Hyper }}}\left(A^{\text {Hypo }}\right)=\mathrm{u}_{\mathfrak{T}_{\text {Hyper }}}\left(\left\{P_{2}, P_{3}\right\}\right)=T_{2}^{\text {Hyper }}=\left\{P_{3}, P_{4}\right\}$.

Informally: in accordance with the nonspecific nature of thyroid dysfunction symptoms, there are symptoms under which patients can possibly be classified as suffering from hypothyroidism with respect to the clinical symptoms which may indicate hyperthyroidism. 


\section{Conclusion and Future Work}

In this paper, beyond classical and recent methods, the authors have proposed a basically new approach for handling imperfect medical data. Its novelty is inherent in the partiality of the applied approximation space. Partial approximation space is a generalization of Pawlakian rough set theory which adequately reflects partial nature of real-life problems. The proposed methods may serve as a basis of computer assistance systems in medicine.

The evaluation of evidence described above is of qualitative nature. Quantifying uncertainty is an important challenge in rough set theory and its generalizations. We will plan to make the model more useful in real applications, first of all, by means of quantifying uncertainty in partial approximation spaces.

Acknowledgements. The publication was supported by the TÁMOP-4.2.2.C$11 / 1 / \mathrm{KONV}-2012-0001$ project. The project has been supported by the European Union, co-financed by the European Social Fund.

\section{References}

1. Ashby, D.: Bayesian statistics in medicine: a 25 year review. Statistics in Medicine 25(21), 3589-3631 (2006)

2. Beynon, M., Curry, B., Morgan, P.: The Dempster-Shafer theory of evidence: an alternative approach to multicriteria decision modelling. Omega 28(1), 37-50 (2000)

3. Bonikowski, Z., Bryniarski, E., Wybraniec-Skardowska, U.: Extensions and intensions in the rough set theory. Information Sciences 107(1-4), 149-167 (1998)

4. Buchanan, B.G., Shortliffe, E.H.: Rule Based Expert Systems: The MYCIN Experiments of the Stanford Heuristic Programming Project. The Addison-Wesley Series in Artificial Intelligence. Addison-Wesley Longman Publishing Co., Inc., Boston (1984)

5. Csajbók, Z., Mihálydeák, T.: A general set theoretic approximation framework. In: Greco, S., Bouchon-Meunier, B., Coletti, G., Fedrizzi, M., Matarazzo, B., Yager, R.R. (eds.) IPMU 2012, Part I. CCIS, vol. 297, pp. 604-612. Springer, Heidelberg (2012)

6. Csajbók, Z.E.: Approximation of sets based on partial covering. In: Peters, J.F., Skowron, A., Ramanna, S., Suraj, Z., Wang, X. (eds.) Transactions on Rough Sets XVI. LNCS, vol. 7736, pp. 144-220. Springer, Heidelberg (2013)

7. Csajbók, Z.: A security model for personal information security management based on partial approximative set theory. In: Ganzha, M., Paprzycki, M. (eds.) Proceedings of the International Multiconference on Computer Science and Information Technology (IMCSIT 2010), Wisła, Poland, October 18-20, vol. 5, pp. 839-845. Polskie Towarzystwo Informatyczne - IEEE Computer Society Press, Katowice (2010)

8. Csajbók, Z.: Simultaneous anomaly and misuse intrusion detections based on partial approximative set theory. In: Cotronis, Y., Danelutto, M., Papadopoulos, G.A. (eds.) Proceedings of PDP 2011, Ayia Napa, Cyprus, February 9-11, pp. 651-655. IEEE Computer Society Press, Los Alamitos (2011) 
9. Csajbók, Z., Mihálydeák, T.: General tool-based approximation framework based on partial approximation of sets. In: Kuznetsov, S.O., Ślęzak, D., Hepting, D.H., Mirkin, B.G. (eds.) RSFDGrC 2011. LNCS (LNAI), vol. 6743, pp. 52-59. Springer, Heidelberg (2011)

10. Csajbók, Z., Mihálydeák, T.: Partial approximative set theory: A generalization of the rough set theory. International Journal of Computer Information Systems and Industrial Management Applications 4, 437-444 (2012)

11. Hassanien, A.E., Abraham, A., Peters, J.F., Schaefer, G.: Rough sets in medical informatics applications. In: Mehnen, J., Saad, M.K.A., Tiwari, A. (eds.) Applications of Soft Computing. AISC, vol. 58, pp. 23-30. Springer, Heidelberg (2009)

12. Keefe, R., Smith, P.: Introduction: Theories of vagueness. In: Keefe, R., Smith, P. (eds.) Vagueness: A Reader, pp. 1-57. MIT Press, Cambridge (1996)

13. Ladenson, P., Kim, M.: Thyroid. In: Goldman, L., Schafer, A.I. (eds.) Goldman's Cecil Medicine, ch. 233. Saunders Elsevier, Philadelphia (2011)

14. Ladenson, P.W., Singer, P.A., Ain, K.B., Bagchi, N., Bigos, S.T., Levy, E.G., Smith, S.A., Daniels, G.H., Cohen, H.D.: American thyroid association guidelines for detection of thyroid dysfunction. Arch. Intern. Med. 160(11), 1573-1575 (2000)

15. Orlowska, E.S.: Logic of vague concepts. Bulletin of the Section of Logic 11(3-4), 115-126 (1982)

16. Ozyılmaz, L., Yıldırım, T.: Diagnosis of thyroid disease using artificial neural network methods. In: Proceedings of ICONIP 2002, Singapore, pp. 2033-2036. Orchid Country Club (2002)

17. Pandey, B., Mishra, R.B.: Knowledge and intelligent computing system in medicine. Comp. in Bio. and Med. 39(3), 215-230 (2009)

18. Patel, V.L., Shortliffe, E.H., Stefanelli, M., Szolovits, P., Berthold, M.R., Bellazzi, R., Abu-Hanna, A.: The coming of age of artificial intelligence in medicine. Artificial Intelligence in Medicine 46(1), 5-17 (2009)

19. Pawlak, Z.: Rough sets. International Journal of Computer and Information Sciences 11(5), 341-356 (1982)

20. Pawlak, Z.: Rough Sets: Theoretical Aspects of Reasoning about Data. Kluwer Academic Publishers, Dordrecht (1991)

21. Pawlak, Z., Skowron, A.: Rough sets: Some extensions. Information Sciences 177, 28-40 (2007)

22. Seising, R.: Fuzzy sets in medicine - historical remarks. In: Valafar, F., Valafar, H. (eds.) METMBS, pp. 31-37. CSREA Press (2004)

23. Seising, R.: From vagueness in medical thought to the foundations of fuzzy reasoning in medical diagnosis. Artificial Intelligence in Medicine 38(3), 237-256 (2006)

24. Skowron, A.: Vague concepts: A rough-set approach. In: De Baets, B., De Caluwe, R., De Tré, G., Fodor, J., Kacprzyk, J., Zadrożny, S. (eds.) Proceedings of EUROFUSE 2004, pp. 480-493. Akademicka Oficyna Wydawnicza EXIT, Warszawa (2004)

25. Spiegelhalter, D.J., Abrams, K.R., Myles, J.P.: Bayesian Approaches to Clinical Trials and Health-Care Evaluation (Statistics in Practice). John Wiley \& Sons (January 2004)

26. Straszecka, E.: Combining uncertainty and imprecision in models of medical diagnosis. Inf. Sci. 176(20), 3026-3059 (2006)

27. Temurtas, F.: A comparative study on thyroid disease diagnosis using neural networks. Expert Syst. Appl. 36(1), 944-949 (2009)

28. Yager, R.R., Kacprzyk, J., Fedrizzi, M. (eds.): Advances in the Dempster-Shafer theory of evidence. John Wiley \& Sons, Inc., New York (1994) 
29. Yao, Y.Y.: On generalizing rough set theory. In: Wang, G., Liu, Q., Yao, Y., Skowron, A. (eds.) RSFDGrC 2003. LNCS (LNAI), vol. 2639, pp. 44-51. Springer, Heidelberg (2003)

30. Zadeh, L.A.: Fuzzy sets. Information and Control 8(3), 338-353 (1965)

31. Zakowski, W.: Approximations in the space $(U, \Pi)$. Demonstratio Mathematica 16(3), 761-769 (1983)

32. Zhu, W.: Topological approaches to covering rough sets. Information Sciences 177(6), 1499-1508 (2007)

33. Zhu, W.: Relationship between generalized rough sets based on binary relation and covering. Information Sciences 179(3), 210-225 (2009) 